\title{
ICI 2012: Pelvic organ prolapse surgery
}

\author{
Christopher Maher
}

\section{(C) ICUD-EAU 2013}

Pelvic organ prolapse (POP) is a common problem affecting up to $50 \%$ of parous women and $6.3 \%$ of women will have undergone a surgical correction for pelvic organ prolapse by the age of 80 [1]. Prolapse surgery is an increasingly important aspect of gynaecological practice because of our ageing population, the decreasing rate of hysterectomy owing to alternative treatments for menorrhagia, and finally decreasing rates of cervical interventions for cervical dysplasia following the introduction of vaccinations for human papilloma virus. Already, prolapse surgery is performed at least as frequently as continence surgery and, the operating and admission times are at least three times greater than for continence surgery. Given the increasing time and resources that will be required for POP surgery in the future it is paramount that we perform effective, durable, cost-effective interventions with minimal morbidity. These articles serve to outline and summarise the information relating to POP surgery reported in the English-language scientific literature after searching PubMed, Medline, the Cochrane library and Cochrane database of systematic reviews, published up to January 2012, with level 1 evidence (randomised controlled trials [RCT] or systematic reviews), level 2 (poor quality RCT, prospective cohort studies) or level 3 evidence (case series or retrospective studies) have been included if level 1 data were lacking. The committee evaluated

On behalf of Committee 15 "Surgical Management of Pelvic Organ Prolapse" from the 5th International Consultation on Incontinence held in Paris, February 2012

This work has been previously published as: Maher C, Baessler K, Barber M, Cheon C, Deitz V, DeTayrac R, Gutman R, Karram M, Sentilhes L (2013) Surgical management of pelvic organ prolapse. In: Abrams, Cardozo, Khoury, Wein, (eds) 5th International Consultation on Incontinence. Health Publication Ltd, Paris, Chapter 15, and modified for publication in International Urogynaecology Journal.

\section{Maher $(\square)$}

Royal Brisbane and Wesley Urogynaecology, University of Queensland, 30 Chaseley Street, Auchenflower, 4067 Brisbane, Queensland, Australia

e-mail: chrismaher@urogynaecology.com.au the literature and made recommendations based on the Oxford grading system summarised as:

Grade A recommendation usually depends on consistent level 1 evidence and often means that the recommendation is effectively mandatory and placed within a clinical care pathway. However, there will be occasions where excellent evidence (level 1) does not lead to a grade A recommendation, for example, if the therapy is prohibitively expensive, dangerous or unethical.

Grade B recommendation usually depends on consistent level 2 and/or 3 studies, or "majority evidence" from RCTs.

Grade C recommendation usually depends on level 4 studies or "majority evidence" from level $2 / 3$ studies or Delphi processed expert opinion.

Grade D "no recommendation possible" would be used where the evidence is inadequate or conflicting and when expert opinion is delivered without a formal analytical process, such as by Delphi.

The recommendations serve as the conclusions of the review and a guide to the status of the current knowledge and future research in POP surgery.

Acknowledgements This publication results from the work of the Committee on Pelvic Organ Prolapse Surgery, part of the 5th International Consultation on Incontinence, held in Paris in February 2012, under the auspices of the International Consultation on Urological Diseases, and enabled by the support of the European Association of Urology.

The authors wish to acknowledge the fine work of previous consultations led by Professor Linda Brubaker.

Conflicts of interest None.

\section{References}

1. Olsen AL, Smith VJ, Bergstrom JO, Colling JC, Clark AL (1997) Epidemiology of surgically managed pelvic organ prolapse and urinary incontinence. Obstet Gynecol 89(4):501-506 